\title{
COMPARTMENT-DIRECTED PHYSICAL EXAMINATION OF THE KNEE CAN PREDICT ARTICULAR CARTILAGE ABNORMALITIES DISCLOSED BY NEEDLE ARTHROSCOPY
}

\author{
ROBERT W. IKE and KENNETH S. O'ROURKE
}

Objective. To determine whether physical examination maneuvers that focus on each knee compartment and assess crepitus at several distinct sites can specifically disclose articular cartilage abnormalities in the compartment being assessed.

Methods. Twenty patients with knee pain were examined before needle arthroscopy. Crepitus was sought from the patellofemoral compartment, medial tibiofemoral compartment, and lateral tibiofemoral compartment. Any crepitus felt in the distal tibia during a tibiofemoral stress maneuver was recorded as transmitted bony crepitus (TBC). Needle arthroscopy assessed articular cartilage ( 5 sites) and both menisci in each knee.

Results. Crepitus by conventional assessment revealed patellar cartilage disruption (69\% sensitive, $50 \%$ specific) and abnormalities of tibiofemoral cartilage (67\% sensitive, $40 \%$ specific) but could not indicate their location. Tibiofemoral crepitus found cartilage disruption in the compartment at a sensitivity of $22 \%$ and a specificity of $100 \%$, and with added tibiofemoral stress, a sensitivity of $65 \%$ and a specificity of $94 \%$ (the one "false positive" had bare bone in the other compartment). TBC was detected in 7 compartments, all of which had focal bare bone on tibial and femoral surfaces; 6 other compartments had tibial bare bone without TBC. Thus, TBC was $54 \%$ sensitive and $100 \%$ specific for tibial bare bone, and $88 \%$ sensitive and $100 \%$ specific for bone-on-bone.

Presented at the 57th National Meeting of the American College of Rheumatology, San Antonio, TX, November 1993. Supported in part by NIH-NIAMS grant 5P60-AR2055.

Robert W. Ike, MD: University of Michigan Medical Center, Ann Arbor; Kenneth S. O'Rourke, MD: Bowman Gray School of Medicine, Wake Forest University, Winston-Salem, North Carolina. Address reprint requests to Robert W. Ike, MD, Division of Rheumatology, 3918 Taubman Center, 1500 East Medical Center Drive, Ann Arbor, MI 48109-0358.

Submitted for publication April 22, 1994; accepted in revised form January 10, 1995
Conclusion. Compartment-directed physical examination of the painful knee can locate and assess the severity of certain articular cartilage abnormalities that are not reliably found by conventional methods. Transmitted bony crepitus is a specific finding for bone-onbone in the compartment being assessed.

Physical examination of the chronically painful knee is a time-honored component of clinical evaluation. However, studies that compare physical findings to the intraarticular anatomy-either suggested by imaging studies or validated by arthroscopic or open inspection-have mainly focused on maneuvers that assess lesions of menisci or ligaments (1-6). Using the physical examination to evaluate hyaline articular cartilage remains a matter of conjecture.

Reproducible, reliable, and inexpensive methods of assessing articular cartilage pathology are desirable as tools for field studies and large-scale clinical trials, as pointed out by Hart et al (7). Crepitus-_" the crackling sound or sensation caused by friction between bone and cartilage. . .'(8)-is recognized as a cardinal feature of osteoarthritis (OA) and is a component of the American College of Rheumatology (ACR) classification criteria for OA of the knee (9); yet, the meaning of this finding remains a matter of debate (10).

As rheumatologists using arthroscopy to assess knee arthritis, we have been impressed that physical examination maneuvers that are meant to find meniscal lesions often suggest the presence of other articular cartilage abnormalities that are later demonstrated by arthroscopy. To determine whether certain modifications of the conventional knee examination-utilizing principles of these maneuvers combined with an assessment for crepitus felt at several distinct sites-can specifically predict abnormalities of articular cartilage, we undertook this prospective study. Our findings suggest that compartment-directed assessment of 


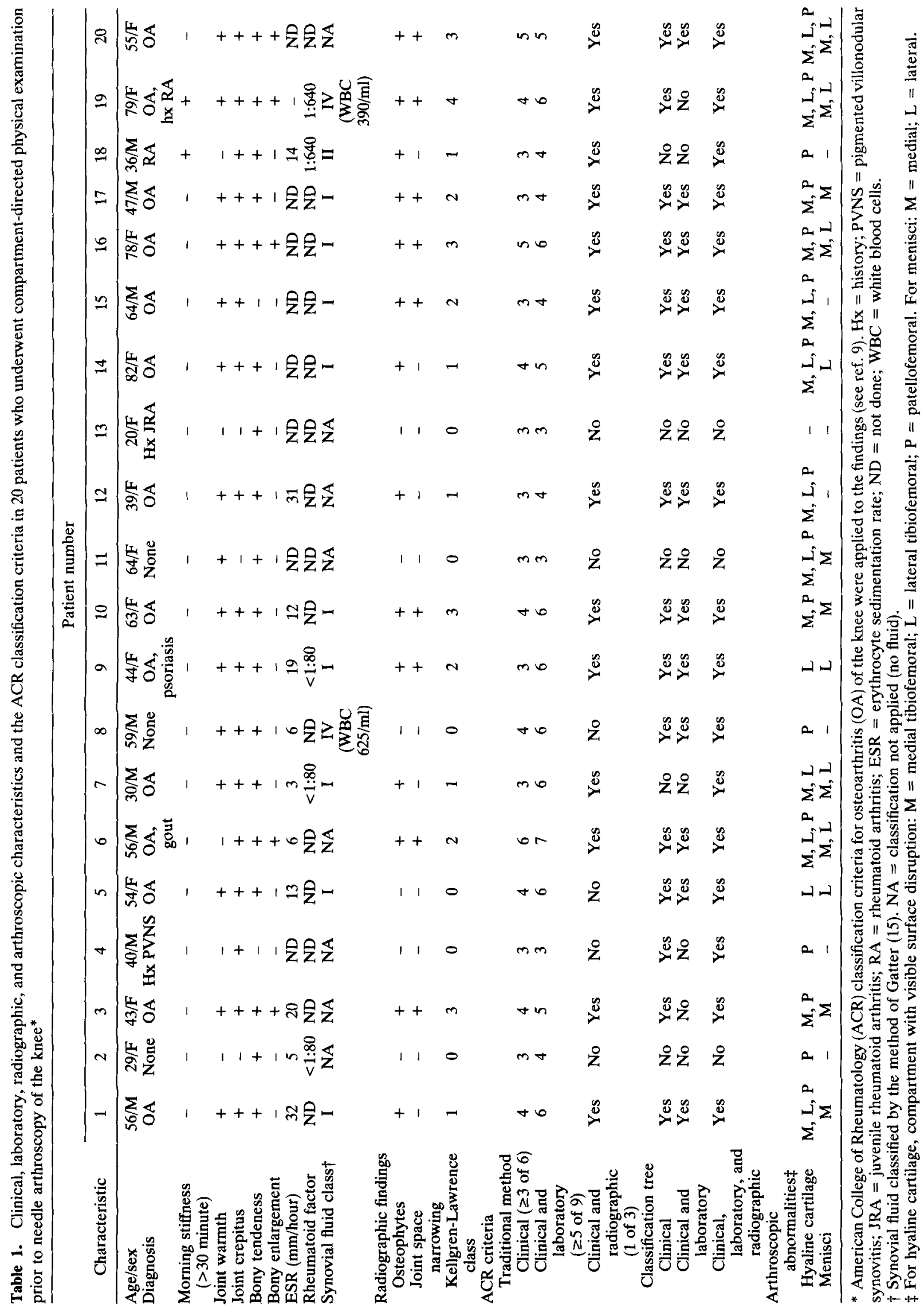


crepitus, when assisted by stressing maneuvers and coupled with palpation for transmission of crepitus, can reliably predict the presence and severity of abnormal articular cartilage in the painful knee.

\section{PATIENTS AND METHODS}

Patient selection. We studied 20 patients from the University of Michigan Rheumatology Clinics who underwent arthroscopy for clinical indications (19 patients) or research purposes ( 1 patient). All patients had knee pain, although the severity varied. Four patients had no other rheumatic disease diagnosis, normal radiographic findings, and knee pain that had not responded to oral analgesics and antiinflammatory agents (all 4 patients), intraarticular glucocorticoids ( 2 patients), or arthroscopic partial meniscectomy ( 1 patient). Eleven patients had primary $O A$ and pain that was refractory to oral analgesics and antiinflammatory agents (all 11 patients), intraarticular glucocorticosteroids ( 9 patients), or arthroscopic partial meniscectomy (1 patient). Five patients had a previously diagnosed inflammatory arthropathy (rheumatoid arthritis [RA] in 2, juvenile RA in 1, gout in 1 , and pigmented villonodular synovitis in 1), although only 1 of these patients (a patient with RA) had signs of joint inflammation at the time of arthroscopy. One RA patient and the patient with gout each had secondary OA.

Data collected were sufficient to judge whether ACR classification criteria for knee OA (9) had been satisfied and included physical assessment, laboratory studies of blood and synovial fluid, and weight-bearing radiographs of the knee. In some patients, rheumatoid factor and erythrocyte sedimentation rate ( 2 of the 3 laboratory tests among the ACR criteria) were not measured, particularly in the absence of any signs of local or systemic inflammation.

Physical examination. We independently examined the symptomatic knee of each patient, and recorded our findings on a standardized form. We noted the presence and size of effusion, palpable warmth, and focal tenderness. We noted any crepitus, and assessed each knee compartment separately during passive motion of the knee, by placing a hand over the medial joint line (medial tibiofemoral compartment), the lateral joint line (lateral tibiofemoral compartment), and the patella (patellofemoral compartment). We assessed each compartment under added stress during passive motion. Our stress maneuvers for the 3 compartments were as follows: medial tibiofemoral compartment, applied varus stress plus external rotation of the tibia; lateral tibiofemoral compartment, applied valgus stress plus internal rotation of the tibia; and patellofemoral compartment, patella pressed dorsally by the examining hand during passive motion. Finally, we determined whether crepitus from either weight-bearing compartment was transmitted to bone by feeling the distal tibia with the hand that was used to rotate the tibia during the stress maneuvers. Any crepitus felt was designated transmitted bony crepitus (TBC). We compared notes from our examinations and reexamined the patient when necessary to agree on the findings.

Arthroscopic technique. We arthroscoped each patient in an outpatient procedure room, using equipment and techniques described previously (11). Patients received mild systemic sedation (alprazolam $1-3 \mathrm{mg}$ orally 30 minutes before the procedure) along with local and intraarticular anesthesia. Each arthroscopy was videotaped.

Assessment of intraarticular pathology. We inspected the articular cartilage surfaces at 5 sites: the medial and lateral tibial plateau, the medial and lateral femoral condyle, and the undersurface of the patella. We graded each lesion according to the system of Noyes and Stabler (12): $1=$ intact surface but subtle abnormality (discoloration or softening), 2 $=$ disruption of surface without exposure of bone, and $3=$ exposed bone. We also judged lesion size by estimating the surface area occupied by each lesion, and then assigning it to 1 of 3 categories: $<25 \%$ of the surface, $25-75 \%$ of the surface, and $>75 \%$ of the surface. The grade and size of the worst lesion seen on any surface provided the status assessment for that surface. We judged each meniscus as normal or abnormal. Assessments made at the time of arthroscopy were validated by review of the tape by one of us (KSO) several months after the last patient in the study was arthroscoped.

Comparisons and statistical analysis. We analyzed physical examination data using univariable techniques, calculating sensitivity and specificity by comparing the results with the criterion standard, arthroscopic assessment of cartilage and menisci. For each operating characteristic thus generated, we calculated $95 \%$ confidence intervals for the result (proportion) as the proportion plus and minus twice its standard error (13).

\section{RESULTS}

Patient characteristics. Table 1 displays characteristics of the 20 patients studied. Only 12 patients had joint effusions, 11 of which were composed of noninflammatory synovial fluid. Only 14 patients showed radiographic OA, with Kellgren-Lawrence classifications (14) as follows: grade 1 (osteophytes only) 5 patients, grade 2 (osteophytes plus minimal-tomild joint space narrowing) 4 patients, grade 3 (osteophytes plus moderate-to-marked joint space narrowing) 4 patients, grade 4 (osteophytes plus obliteration of joint space and deformity) 1 patient. However, all patients fulfilled the ACR OA classification criteria by at least 1 method.

Of 15 patients who had abnormal hyaline cartilage affecting weight-bearing surfaces, 14 fulfilled $\geq 4$ sets of ACR criteria. Four of the other 5 patients fulfilled $\leq 3$ sets of ACR criteria. Thus, fulfillment of at least 4 sets of ACR criteria was $93 \%$ sensitive and $80 \%$ specific for abnormal weight-bearing hyaline cartilage in this group of patients.

Compartmental crepitus. Figure 1 displays the relationship between crepitus and articular cartilage abnormalities for each of the 60 knee compartments assessed.

Patellofemoral compartment findings. For the patellofemoral compartment (Figure 1A), we noted 
A

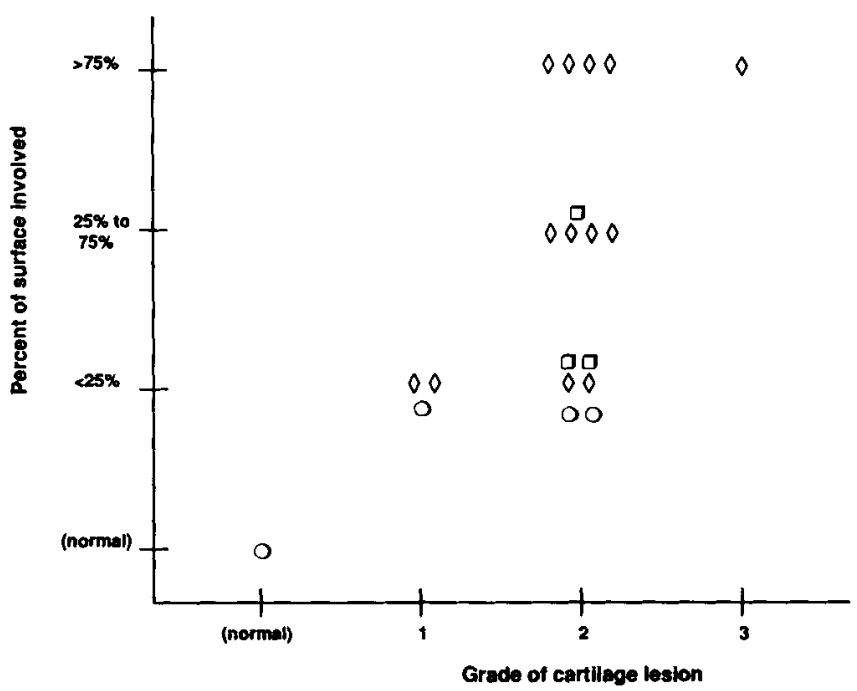

$\mathrm{C}$

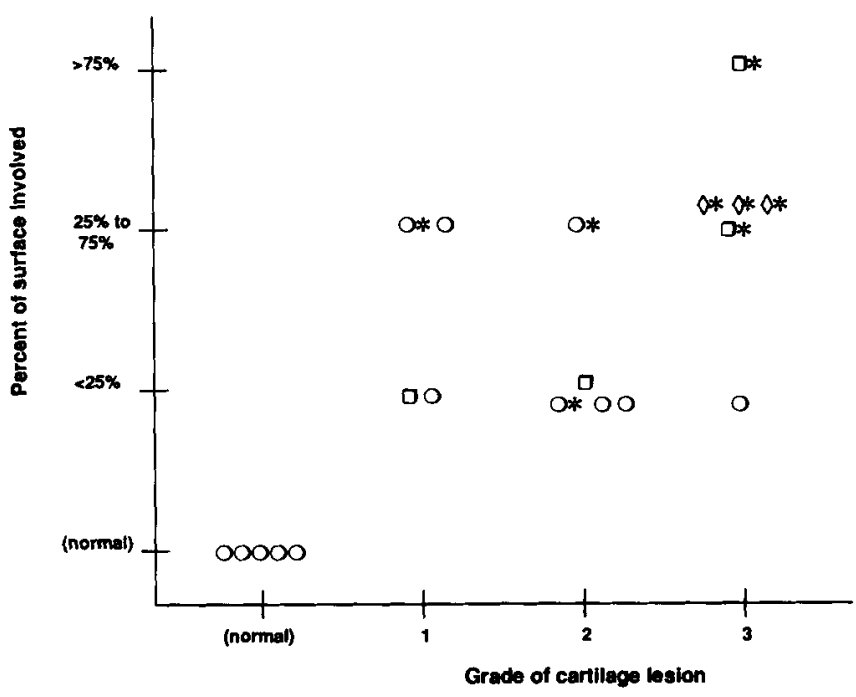

crepitus in 13 knees, 11 of which had patellar cartilage abnormalities of grade $\geq 2$. The 2 "false positives" each had abnormal tibiofemoral articular cartilage, with one knee showing an abnormal meniscus and grade 3 articular cartilage abnormalities (bare bone), while the other had an abnormal meniscus and surface disruption in both tibiofemoral compartments. With added stress, we noted patellofemoral crepitus in 3 other knees, all with smaller lesions than the 11 that were detected without stress. Two knees had patellar cartilage surface disruption but no crepitus, even with added stress. These 2 "false negatives" had small

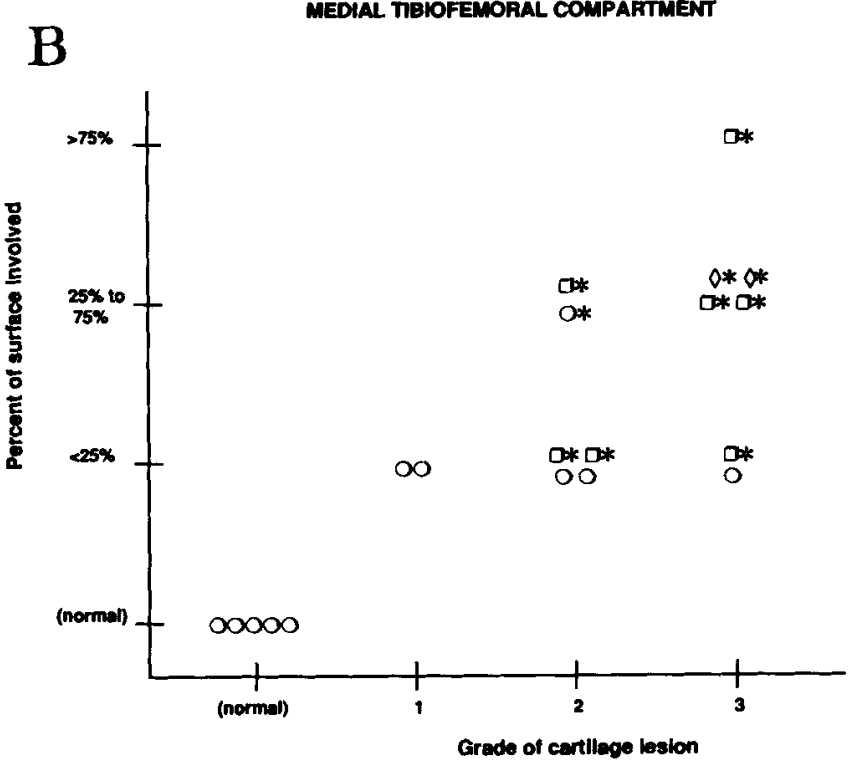

Figure 1. Relationship between crepitus as detected by compartment-directed physical examination, and status of articular cartilage in each compartment as shown by needle arthroscopy. A, Patellofemoral compartment; B, medial tibiofemoral compartment; C, lateral tibiofemoral compartment. $\diamond=$ crepitus with passive range of motion; $\square=$ crepitus only with applied stress; $O=$ no crepitus felt; ${ }^{*}=$ abnormal meniscus at arthroscopy.

lesions ( $<25 \%$ of the surface area) and no other crepitus-generating compartments.

Patellofemoral crepitus did not reliably indicate tibiofemoral cartilage pathology. Of 16 knees with patellofemoral crepitus, 13 had surface disruption in at least 1 tibiofemoral compartment, 11 of which had patellofemoral surface disruption. The other 3 had normal tibiofemoral articular cartilage. Two knees had abnormal tibiofemoral cartilage but no patellofemoral crepitus.

Medial tibiofemoral compartment findings. We analyzed findings from each weight-bearing compart- 

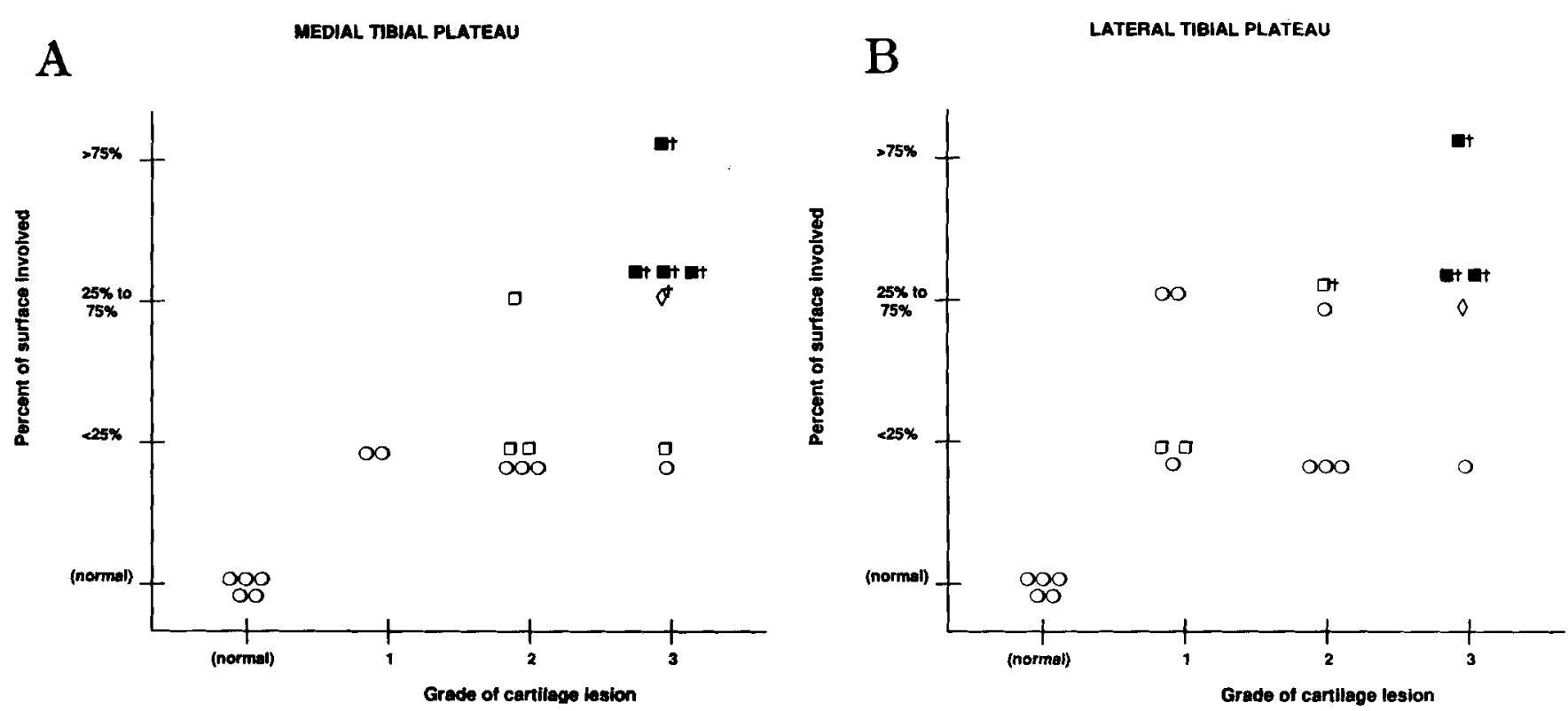

Figure 2. Relationship between transmitted bony crepitus and status of tibial cartilage in the corresponding compartment. A, Medial tibial plateau; B, lateral tibial plateau. $\diamond=$ crepitus with passive range of motion; $\square=$ crepitus only with applied stress; $\mathbf{\square}=$ crepitus transmitted to distal tibia (transmitted bony crepitus); $O=$ no crepitus felt; $\dagger=$ grade 3 (bare bone) lesion, adjacent femoral condyle.

ment separately. We felt crepitus from the medial tibiofemoral compartment in only 2 knees, but with stress elicited crepitus from 7 others (Figure 1B). Both knees with easily detected medial tibiofemoral crepitus had focal tibial bare bone and abnormal medial menisci. The 7 medial compartments generating crepitus only with stress had some tibiofemoral cartilage surface disruption (4 had grade 3, 3 had grade 2) and all had abnormal medial menisci. For cartilage lesions in the medial compartments of these 7 knees, 6 were smaller or the same size as the lesions in the 2 knees with "unstressed" medial crepitus. Three compartments had some disrupted articular cartilage but made no crepitus; one of these compartments had an abnormal meniscus. However, there were no "false positives" for the medial compartment.

Lateral tibiofemoral compartment. We felt crepitus from the lateral tibiofemoral compartment in 3 knees, and with stress elicited crepitus from 4 others (Figure 1C). The 3 knees with easily detected lateral crepitus each had focal tibial bare bone and abnormal lateral menisci. Of 4 lateral compartments generating crepitus only with stress, 3 had some tibiofemoral cartilage surface disruption ( 2 had grade 3,1 had grade 2) and 2 had abnormal lateral menisci. In 2 of these 3 knees, lateral compartment lesions were the same size or smaller than the lesions in the 2 knees with "unstressed" lateral crepitus. Four compartments con- tained some disrupted articular cartilage, but did not generate crepitus; 2 of these compartments had an abnormal meniscus. The only "false positive" compartment featured extensive articular cartilage abnormalities in patellofemoral and medial tibiofemoral compartments along with an abnormal medial meniscus; we had detected crepitus without additional stress from these other compartments.

Transmitted bony crepitus. We detected crepitus transmitted to the distal tibia when assessing one of the weight bearing compartments much less frequently than conventional and compartment-directed crepitus. Presuming that crepitus in the distal tibia would come from the proximal tibial surface, we analyzed the relationship of this finding to the status of the tibial plateau cartilage in the weight-bearing compartment being assessed (Figures $2 \mathrm{~A}$ and $\mathrm{B}$ ). We detected TBC from 7 compartments in 6 knees. All 7 compartments had bare bone occupying $>25 \%$ of the tibial plateau, accompanied by focal bare bone of the adjacent femoral condyle. Five other compartments in 4 knees had focal tibial bare bone but did not generate TBC. Compared with the 7 compartments that did, cartilage lesions were smaller ( 3 occupied $<25 \%$ of the surface, while 2 occupied $25-75 \%$ of the surface) and were accompanied by less severe articular cartilage lesions of the adjacent femoral condyle, with only 1 grade- 3 lesion. Thus, TBC from a weight-bearing compartment 
seemed to reliably indicate "bone-on-bone" in that compartment.

Operating characteristics of examination maneuvers. The operating characteristics of the various compartment-directed examination findings we analyzed for predicting arthroscopically disclosed articular cartilage abnormalities are shown in Table 2. Crepitus found by the examining hand resting on the patella, as knee crepitus is usually assessed, predicted patellar cartilage disruption more than two-thirds of the time when it was present, but was only 50\% specific for this abnormality; weight-bearing cartilage lesions were predicted with similar sensitivity and specificity by crepitus elicited in this manner. Placing stress on the patellofemoral compartment while eliciting crepitus increased the sensitivity somewhat both for patellar and tibiofemoral lesions, but did not affect specificity. However, specific detection of weightbearing cartilage lesions required compartmentdirected assessment and added stress.

The medial tibiofemoral compartment was more reliably assessed by directed maneuvers than was the lateral compartment. Feeling for crepitus at the joint line of a compartment during passive motion proved to be an insensitive method for predicting cartilage lesions, since it was positive in only a few compartments with surface disruption ( $15 \%$ medial, $27 \%$ lateral) or abnormal menisci (20\% medial, $38 \%$ lateral). However, adding stress to the compartment being assessed brought crepitus to the fore that disclosed many more cartilage and meniscal lesions without appreciably affecting specificity, which remained $100 \%$ in the medial compartment but fell slightly in the lateral compartment due to a "false positive" in which advanced lesions in the other compartments likely generated the crepitus we felt.

Finally, "bare bone" was reliably predicted by finding crepitus transmitted to the distal tibia from the weight-bearing compartment assessed while being stressed. While this maneuver was not especially sensitive for tibial bare bone lesions (medial 58\%, lateral $60 \%$ ), the finding was $100 \%$ specific and was dependent on the size of the tibial lesion and the presence of bare bone on the adjacent femoral condylar surface. Indeed, this maneuver predicted all but one ( $80 \%$ sensitive for medial, $100 \%$ for lateral) of the compartments with "bone-on-bone" potential (bare bone lesions, however small, on tibial and femoral surfaces) and remained $100 \%$ specific.

For all operating characteristics of these maneuvers, confidence intervals were quite broad because of the small number of patients studied. Speci- ficities determined for maneuvers that found "bare bone" lesions showed the narrowest confidence limits; hence, these tests could maintain their specificity with wider testing, and thus prove useful by "ruling in" the target disorder (here, "bare bone") when positive (13).

\section{DISCUSSION}

We undertook this study to see whether the physical examination of the knee we had come to use on patients being considered for arthroscopy was yielding reliable information about articular cartilage. Our examination had evolved to incorporate maneuvers commonly used to seek meniscal lesions, such as palpation of the joint line and a search for vibrations arising from each weight-bearing compartment when stressed by rotation of the tibia. Several tests suggest meniscal pathology when tibial rotation causes pain or when palpation finds tenderness or clicks (16). Our "stress maneuver" for the tibiofemoral compartment combines the McMurray test with precepts underlying Bohler's sign. McMurray described extending the knee from maximal flexion-rotating the tibia externally to stress the medial compartment and internally to stress the lateral compartment-while palpating the respective joint line for a click that would indicate a torn meniscus therein (17). Bohler's sign suggests meniscal pathology when varus stress (medial tear) or valgus stress (lateral tear) causes pain (16). These maneuvers, when combined, often produced crepitus we had missed on routine examination; corresponding articular cartilage lesions seemed to be the rule in those knees we later arthroscoped. Data from the physical examination and arthroscopy of the 20 patients in this study support our suspicion that compartmentdirected physical examination of the knee can predict the location and severity of certain articular cartilage abnormalities.

Only 3 previous studies have prospectively compared physical examination findings to directly inspected intraarticular pathology, and each evaluated signs considered specific for meniscal lesions $(1,3,6)$. McMurray's sign was found to be the most specific of the conventional signs (95\% in 2 studies), but it was relatively insensitive (58\% [ref. 3] and $29 \%$ [ref. 6]). Anderson and Lipscomb described a maneuver which added stress during passive extension that proved even more specific (99\%) (3). None of these 3 studies commented on the condition of weight-bearing articular cartilage. We found abnormal menisci in 13 of the 20 knees we evaluated, and had elicited crepitus from most compartments that contained abnormal menisci. 


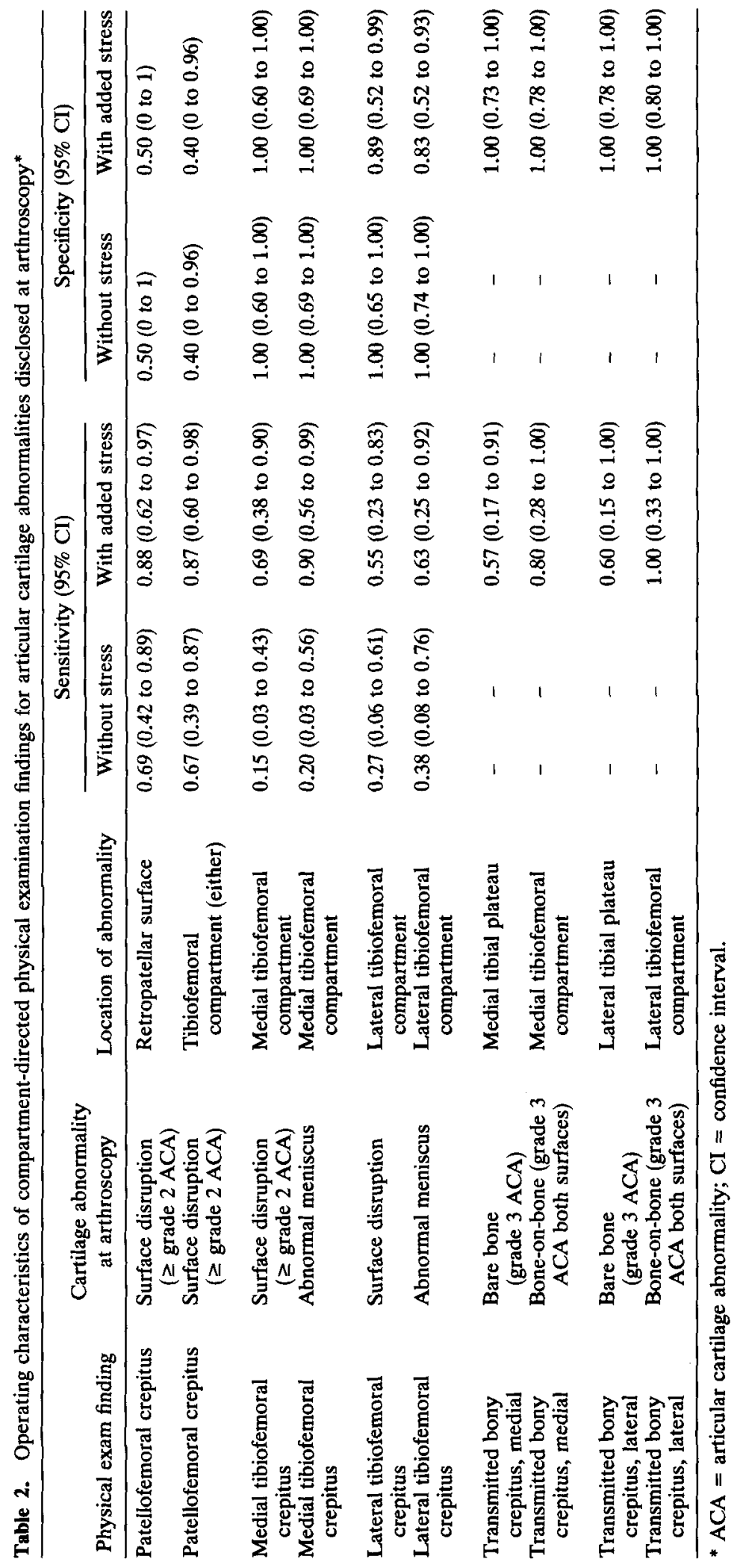


Taken without consideration of hyaline cartilage pathology, the operating characteristics of our findings for abnormal menisci compare favorably with the findings of these previous studies. However, we found crepitus and abnormal menisci only when the cartilage surface was disrupted. Also, we saw abnormal menisci in several "silent" compartments. Thus, we consider crepitus to indicate cartilage surface disruption rather than meniscal pathology.

When we felt crepitus from the tibiofemoral compartment under investigation and from the distal tibia, we always found patches of bare bone on the tibial plateau of that compartment and on the adjacent femoral condyle, although the lesions were not always large. We suspect that crepitus arising from a disrupted joint surface is dampened by residual underlying cartilage, but that exposed bone on the tibial surface permits these vibrations to be transmitted distally, where they can be felt in the tibia. Since we felt transmitted crepitus only when both surfaces comprised some bare bone, the more intense vibration of "bone-on-bone" movement is probably necessary to make noise loud enough to be felt in the distal tibia.

Our attempts to deduce bony pathology from transmitted vibrations are hardly the first such efforts. Laennec, in the appendix to his treatise on medical auscultation (18), described the findings of Lisfranc, who deduced the presence of fractures in long bones by auscultation of the bone ends. Hugh Little differentiated fine crepitus from coarse crepitus, the latter arising "from damaged cartilagenous or bony surfaces rubbing on one another (and) palpable throughout the bones from which it arises"; he also indicated that "One can deduce the origin of crepitus by palpating the bones during active movement and finding the common articulation" (19). Paice mentioned "eburnation crepitus" as a finding that is "both heard and felt, occurring when the cartilage has been destroyed and the two bony surfaces are in contact" (20). Other modern discussions of the musculoskeletal physical examination, however, do not mention these phenomena.

Besides describing knee examination maneuvers that had not heretofore been used to assess cartilage, we sought to determine the precision of these findings. We found all manuevers examining the tibiofemoral compartments to be rather specific and, with applied stress, reasonably sensitive in this group of patients. However, our analytic methods sometimes fell short of standards that have been set forth for such an exercise (21). Since we conducted the "test" (physical finding) and also assessed the "gold standard" (arthroscopy), we forfeited the analytic rigor that would arise from independent assessment. Ideally, the arthroscopic examination and formal grading of intraarticular findings, whether done at the time of arthroscopy or at the time of review of the videotape, would have been conducted by someone unaware of the physical findings. By analyzing videotape several months after arthroscopy (and physical examination), we hoped to reduce some of this potential bias. However, the physical examination could have biased the arthroscopy itself, with areas that seemed abnormal on examination being given closer scrutiny at arthroscopy, thereby enhancing the chances of finding intraarticular pathology. Because we each examined our patients only once or twice on the same day (and only a few minutes apart) and chose to reach consensus on each finding rather than record any initial discrepancies between our two examinations, we cannot comment on the reproducibility or observer variation of these findings. As more physicians become aware of the physical examination maneuvers we have described, studies designed to remedy these 2 major shortcomings of our initial analysis should be feasible.

The reliability and appropriateness of crepitus as a screening or diagnostic sign have been questioned $(10,22)$. Cushnaghan et al found that 5 observers examining $8 \mathrm{OA}$ patients twice within a few hours agreed well with themselves and with each other about the presence of tibiofemoral crepitus, but less so about patellofemoral crepitus (23). However, when Hart et al found knee crepitus to be an uncommon sign (7\% of the 41 middle-aged women examined) about which their 2 investigators did not always agree $(65 \%$ concordance), they excluded it from their study of clinical signs of $O A$ in a larger population (7). Jones and 4 colleagues, who examined both knees of 49 patients with a wide range of pathology, found that they, as 5 examiners, seldom agreed on physical signs; however, their intraobserver variation was lowest for tibiofemoral crepitus (22). Since examiners in each of these studies did not elicit findings-including crepitus--by a specific protocol, considerable interobserver variation is not unexpected. Eliciting crepitus by placing a hand on the "knee" (patella) during passive motion proved insensitive and nonspecific in our studies as well. We agree with Cushnaghan et al that "more clearly defined protocols should be devised for eliciting specific physical signs" (23). Since assessment of the tibiofemoral compartment seems to be among the more repeatable physical signs in $\mathrm{OA}$, we can hope 
that the methods we described will prove reproducible on wider testing.

There are several uses for the additional physical signs we have described. By finding crepitus in the weight-bearing compartment of the knee in a patient with otherwise unexplained knee pain, the clinician can diagnose OA as part of the problem. In the patient with established $\mathrm{OA}$, detecting TBC could confirm progression to "advanced" disease, regardless of radiographic features. This finding could dissuade recommendation for arthroscopic surgery in patients with knee OA, who are far less likely to improve following "debridement" if bare bone is present (24). Although therapies that might modify OA cartilage are still experimental or in development (25), reproducible means of assessing cartilage non-invasively would be useful in clinical tests of such agents. Small accelerometers (microphones) can record and quantitate crepitus, and could be used to enhance the precision of the examiner's hand (26). Whether investigators conducting field studies of $\mathrm{OA}$ might benefit from compartment-directed crepitus as assessment tools remains to be determined. Disrupted cartilage surfaces that produce crepitus may not always denote lesions that are destined to progress to more typical OA. Bergquist et al found that knee crepitus was so common in a group of elderly patients that the finding would not be valid or reliable for population studies of OA (27). Use of a directed examination, rather than the conventional method of assessing crepitus (that mainly judges the patellofemoral compartment), could serve otherwise, as would restricting assessment to those measures (transmitted bony crepitus) that reveal severe OA lesions.

\section{ACKNOWLEDGMENT}

We thank M. Anthony Schork, PhD, for assistance with the biostatistical analysis.

\section{REFERENCES}

1. Noble J, Erat K: In defence of the meniscus. J Bone Joint Surg [Br] 62:7-11, 1980

2. Katz JW, Fingeroth RJ: The diagnostic accuracy of ruptures of the anterior cruciate ligament comparing the Lachman test, the anterior drawer sign, and the pivot shift test in acute and chronic knee injuries. Am J Sports Med 14:88-91, 1986

3. Anderson AF, Lipscomb AB: Clinical diagnosis of meniscal tears: description of a new manipulative test. Am J Sports Med 14:291-293, 1986

4. Bonamo JJ, Shulman G: Double contrast arthrography of the knee: a comparison to clinical diagnosis and arthroscopic findings. Orthopedics 11:1041-1046, 1988

5. Hardaker WT Jr, Garrett WE Jr, Bassett FH III: Evaluation of acute traumatic hemarthrosis of the knee joint. South Med J 83:640-644, 1990

6. Fowler PJ, Lubliner JA: The predictive value of five clinical signs in the evaluation of meniscal pathology. Arthroscopy $5: 184-186,1989$

7. Hart DJ, Spector TD, Brown P, Wilson P, Doyle DV, Silman AJ: Clinical signs of early osteoarthritis: reproducibility and relation to X-ray changes in 541 women in the general population. Ann Rheum Dis 50:467-470, 1991

8. Brown L, ed: New Shorter Oxford English Dictionary. Oxford, Clarendon Press, 1993

9. Altman R, Asch E, Bloch D, Bole G, Borenstein D, Brandt K, Christy W, Cooke TD, Greenwald R, Hochberg M, Howell D, Kaplan D, Koopman W, Longley S III, Mankin H, McShane DJ, Medsger T Jr, Meenan R, Mikkelsen W, Moskowitz R, Murphy W, Rothschild B, Segal M, Sokoloff L, Wolfe F: Development of criteria for the classification and reporting of osteoarthritis: classification of osteoarthritis of the knee. Arthritis Rheum 29:1039-1049, 1986

10. McAlindon T, Dieppe PA: Osteoarthritis: definitions and criteria. Ann Rheum Dis 48:531-532, 1989

11. Ike RW, O'Rourke KS: Detection of intraarticular abnormalities in osteoarthritis of the knee: a pilot study comparing needle arthroscopy with standard arthroscopy. Arthritis Rheum 36: 1353-1363, 1993

12. Noyes FR, Stabler CL: A system for grading articular cartilage lesions at arthroscopy. Am J Sports Med 17:505-513, 1989

13. Sackett DL, Haynes RB, Tugwell P: Clinical Epidemiology: A Basic Science for Clinical Medicine. Second edition. Boston: Little, Brown and Company, 1991

14. Kellgren JH, Lawrence JS: Radiological assessment of osteoarthritis. Ann Rheum Dis 16:494-501, 1957

15. Gatter RA: A Practical Handbook of Joint Fluid Analysis. Philadelphia, Lea \& Febiger, 1984

16. Tria AJ, Klein KS: An illustrated guide to the knee. New York: Churchill Livingstone, 1992

17. McMurray TP: The semilunar cartilages. Br J Surg 29:407-414, 1949

18. Laennec RTH: A Treatise on the Diseases of the Chest and on Mediate Auscultation. Translated by J Forbes. London, Underwood, 1848

19. Little H: The abnormal joint. In, The Rheumatological Physical Examination. Edited by H Little. Orlando, Grune \& Stratton, 1986

20. Paice E: Principles of examination of the musculoskeletal system. In, Oxford Textbook of Rheumatology. Edited by PJ Maddison, DA Isenberg, P Wood, DJ Glass. Oxford: Oxford University Press, 1993

21. Sackett DL: A primer on the precision and accuracy of the clinical examination. JAMA 267:2638-2644, 1992

22. Jones A, Hopkinson N, Pattrick M, Berman P, Doherty M: Evaluation of a method for clinically assessing osteoarthritis of the knee. Ann Rheum Dis 51:243-245, 1992

23. Cushnaghan J, Cooper C, Dieppe P, Kirwan J, McAlindon T, McCrae F: Clinical assessment of osteoarthritis of the knee. Ann Rheum Dis 49:768-770, 1990

24. Ogilvie-Harris DJ, Fitsialos DP: Arthroscopic management of the degenerative knee. Arthroscopy 7:151-157, 1991

25. Creamer P, Dieppe PA: Novel drug strategies for osteoarthritis. J Rheumatol 20:1461-1463, 1993

26. McCoy GF, McCrea JD, Beverland DE, Kernohan WG, Mollan RA: Vibration arthrography as a diagnostic aid in diseases of the knee: a preliminary report. J Bone Joint Surg [Br] 69:288-293, 1987

27. Bergquist SR, Hannan MT, Neuhauser KB, Wieland DA, Felson DT: Lack of validity and reliabilty of physical exam components of the American College of Rheumatology osteoarthritis diagnostic criteria (abstract). Arthritis Rheum 36 (suppl 9):S58, 1993 